\title{
Quantum Fisher Information of N-Qubit W And GHZ Superposition State Under Nonlocal Operation
}

\section{Yan Li ( $\nabla$ li8989971@163.com )}

Taiyuan Normal University https://orcid.org/0000-0003-0628-6153

\section{Research Article}

Keywords:

Posted Date: February 11th, 2022

DOI: https://doi.org/10.21203/rs.3.rs-1181326/v1

License: (a) (i) This work is licensed under a Creative Commons Attribution 4.0 International License. Read Full License

Version of Record: A version of this preprint was published at The European Physical Journal Plus on April 1st, 2022. See the published version at https://doi.org/10.1140/epjp/s13360-022-02701-y. 


\title{
Quantum Fisher information of $N$-qubit W and GHZ superposition state under nonlocal operation
}

\author{
Yan $\operatorname{Li}^{12 a}$ \\ 1 Department of Physics, Taiyuan Normal University, Jinzhong, 030619, China \\ 2 Institute of Computational and Applied Physics, Taiyuan Normal University, Jinzhong, 030619, China
}

Received: date / Revised version: date

\begin{abstract}
From the perspective of nonlocal operation, we investigate the Quantum Fisher information of $N$-qubit $\mathrm{W}$ and GHZ superposition state. Taking advantage of the general Ising model and Lipkin-Meshkov-Glick model, we analytically present the QFI of $N$-qubit superposition state. Evidently the traditional fixed QFI is changed and largely increased. As an paradigm, we numerically study the QFI of 3-qubit superposition state with respect to ratio $\alpha$ and interaction strength $\varepsilon$, and find the trends of QFI in both models are similar. There always exists a turning point for QFI and the $\alpha$ is around 0.7. Interestingly, for the interaction strength $\varepsilon=1$ in Ising model, the well-known Heisenberg limit is almost achieved for all $\alpha$. Moreover, under the same interaction strength, we compare the QFI of $N$-qubit superposition state in both models. With the increasing qubits involved, the $\alpha$ for maximal QFI in Ising model is changed from close to 1 to the middle region, while it is different in Lipkin-Meshkov-Glick model and the $\alpha$ is close to 1.
\end{abstract}

\section{Introduction}

Quantum Fisher information (QFI) [1-4], as a quantum version of the classical Fisher information [5,6], becomes more and more important in exploring the fundamentals of quantum mechanics, such as quantum metrology [7-9], quantum entanglement $[10,11]$, quantum phase transition [12-16], and etc. As dictated by Cramer-Rao lower bound [17, 18], the QFI can be used to set the ultimate limit to precision measurement and hence facilitates the quantum-enhanced metrological schemes [19,20]. Besides, it also plays an increasingly vital role in multipartite entanglement detection [21-25] and entanglement structure classification $[26,27]$. Several experiments have successfully performed entanglement detection via QFI [28-31, 15, 32,33]. In particular, it is recently employed to witness entanglement in an interactive quantum system [34], which opens up a new direction to explore QFI, especially for those experiment-relevant state.

In this paper, we study the QFI of $N$-qubit $\mathrm{W}$ and GHZ superposition state under the non-local operation. Although the state has been studied by many authors [35-38], it is researched under local operation and few to be addressed by nonlocal operation [39]. Here we investigate it in the Ising model [40] and Lipkin-Meshkov-Glick (LMG) model [41]. We analytically present the QFI of $N$-qubit superposition state and analysis it with respect to ratio $\alpha$ and interaction strength $\varepsilon$. The results show that the QFI of superposition state is increased in both models and is beneficial to quantum metrology. In the case of 3-qubit superposition state, an interesting phenomenon is discovered that the conventional Heisenberg Limit (the inverse of the number of qubits employed) is almost achieved for all $\alpha$ as $\varepsilon=1$ in Ising model. We also find that there always exists a turning point for QFI in both models and the $\alpha$ is around 0.7. Besides, we compare the QFI of $N$-qubit superposition state under the same $\varepsilon$, and the maximal QFI with respect to ratio $\alpha$ is discussed.

The paper is structured as follows. In Section. 2, we briefly review the $N$-qubit W and GHZ superposition state and the formula to calculate QFI of a general quantum state under two-body nonlocal operation. Then in Section. 3 we respectively study the QFI of $N$-qubit superposition state in Ising model and LMG model. Specially, we in detail investigate the QFI of 3-qubit superposition state. Finally, we summarize our results in Section. 4.

\section{Model and Method}

Nonlocal operation means a many-body interaction process and usually it is difficult to be tackled. For simplicity we take twobody interaction as our subject of study,

$$
H=H_{0}+H_{1},
$$

\footnotetext{
a li8989971@163.com
} 
where $H_{0}=\sum_{i=1}^{N} \frac{\mu_{i}}{2} \sigma_{m}^{(i)}$ and $H_{1}=\sum_{i, j=1}^{N} \frac{V_{i j}}{4} \sigma_{n}^{(i)} \sigma_{n}^{(j)}$ represent the local operation and nonlocal operation, respectively. Here $\mu_{i}$ denotes inhomogeneous linear couplings and $V_{i j}=V_{j i}, \sigma_{n}^{(i)}=\sigma^{(i)} \cdot n$ is the pauli matrix for the $i$ th particle and $n$ is a vector. The $N$-qubit W and GHZ superposition state is expressed as

$$
\left|\psi_{N}\right\rangle=\alpha\left|W_{N}\right\rangle+\beta\left|\mathrm{GHZ}_{N}\right\rangle
$$

where the coefficients satisfiy $|\alpha|^{2}+|\beta|^{2}=1$. Assuming the qubit system is denoted by $|0\rangle$ and $|1\rangle$, the $N$-qubit W state and $N$-qubit GHZ state are given by

$$
\left|W_{N}\right\rangle=\frac{1}{\sqrt{N}}\left(\left|0^{\otimes(N-1)}\right\rangle|1\rangle+\sqrt{N-1}\left|W_{N-1}\right\rangle|0\rangle\right)
$$

and

$$
\left|\mathrm{GHZ}_{N}\right\rangle=\frac{1}{\sqrt{2}}\left(\left|0^{\otimes N}\right\rangle+\left|1^{\otimes N}\right\rangle\right)
$$

According to the formula of QFI for pure state under a unitary transformation $e^{-i \hat{H} \theta}[4], F_{Q}=4 \Delta^{2} H$, we have

$$
F_{Q}=\nu_{0}^{2}+\nu_{1}^{2}+\nu_{2}^{2}
$$

where $\nu_{0}^{2}=4 \Delta^{2} H_{0}, \nu_{1}^{2}=4\left(\left\langle\left\{H_{0}, H_{1}\right\}\right\rangle-2\left\langle H_{0}\right\rangle\left\langle H_{1}\right\rangle\right)$ and $\nu_{2}^{2}=4 \Delta^{2} H_{1}$ separately denote the local QFI, covariant QFI and nonlocal QFI. With respect to a general quantum state and the nonlocal operation Eq. (1), they are specifically given by [42]

$$
\begin{aligned}
v_{0}^{2} & =\sum_{i=1}^{N} \mu_{i}^{2}\left(1-\left\langle\sigma_{m}^{(i)}\right\rangle^{2}\right)+\sum_{\substack{i, j=1 \\
i \neq j}}^{N} \mu_{i} \mu_{j}\left(\left\langle\sigma_{m}^{(i)} \sigma_{m}^{(j)}\right\rangle-\left\langle\sigma_{m}^{(i)}\right\rangle\left\langle\sigma_{m}^{(j)}\right\rangle\right) \\
v_{1}^{2} & =2 \sum_{\substack{i, j=1 \\
i \neq j}}^{N} \mu_{i} V_{i j}\left(\mathbf{m} \cdot \mathbf{n}\left\langle\sigma_{n}^{(j)}\right\rangle-\left\langle\sigma_{n}^{(i)} \sigma_{n}^{(j)}\right\rangle\left\langle\sigma_{m}^{(i)}\right\rangle\right)+ \\
& +\sum_{\substack{i, j, k=1 \\
i \neq j \neq k}}^{N} \mu_{k} V_{i j}\left(\left\langle\sigma_{n}^{(i)} \sigma_{n}^{(j)} \sigma_{m}^{(k)}\right\rangle-\left\langle\sigma_{n}^{(i)} \sigma_{n}^{(j)}\right\rangle\left\langle\sigma_{m}^{(k)}\right\rangle\right)
\end{aligned}
$$

and

$$
\begin{aligned}
v_{2}^{2} & =\sum_{\substack{i, j=1 \\
i \neq j}}^{N} \frac{V_{i j}^{2}}{2}\left(1-\left\langle\sigma_{n}^{(i)} \sigma_{n}^{(j)}\right\rangle^{2}\right)+ \\
& +\sum_{\substack{i, j, k=1 \\
i \neq j \neq k}}^{N} V_{i j} V_{j k}\left(\left\langle\sigma_{n}^{(i)} \sigma_{n}^{(k)}\right\rangle-\left\langle\sigma_{n}^{(i)} \sigma_{n}^{(j)}\right\rangle\left\langle\sigma_{n}^{(j)} \sigma_{n}^{(k)}\right\rangle\right)+ \\
& \sum_{\substack{i, j, k, l=1 \\
i \neq j \neq k \neq l}}^{N} \frac{V_{i j} V_{k l}}{4}\left(\left\langle\sigma_{n}^{(i)} \sigma_{n}^{(j)} \sigma_{n}^{(k)} \sigma_{n}^{(l)}\right\rangle-\left\langle\sigma_{n}^{(i)} \sigma_{n}^{(j)}\right\rangle\left\langle\sigma_{n}^{(k)} \sigma_{n}^{(l)}\right\rangle\right) .
\end{aligned}
$$

The QFI can therefore be obtained through the calculation of first-order moment of the interactive qubits, i.e., $\left\langle\sigma_{n}^{(i)}\right\rangle,\left\langle\sigma_{n}^{(i)} \sigma_{n}^{(j)}\right\rangle$ and etc. In the following we will focus on the QFI of $N$-qubit W and GHZ superposition state in Ising model and LMG model, and further analyze its performance in quantum metrology.

\section{Results}

\subsection{QFI of $N$-qubit superposition state in Ising model}

We first consider the $N$-qubit superposition state is operated under the homogeneous nearest-neighbor nonlocal operation, i.e., $\mathbf{m} \cdot \mathbf{n}=1, \mu_{i}=1$ and $V_{i j}=\varepsilon \frac{\delta_{i, j-1}+\delta_{i, j+1}}{2}, \sigma_{n}^{(i)}=a \sigma_{x}^{(i)}+b \sigma_{y}^{(i)}+c \sigma_{z}^{(i)}$. It is usually difficult to be managed because there 

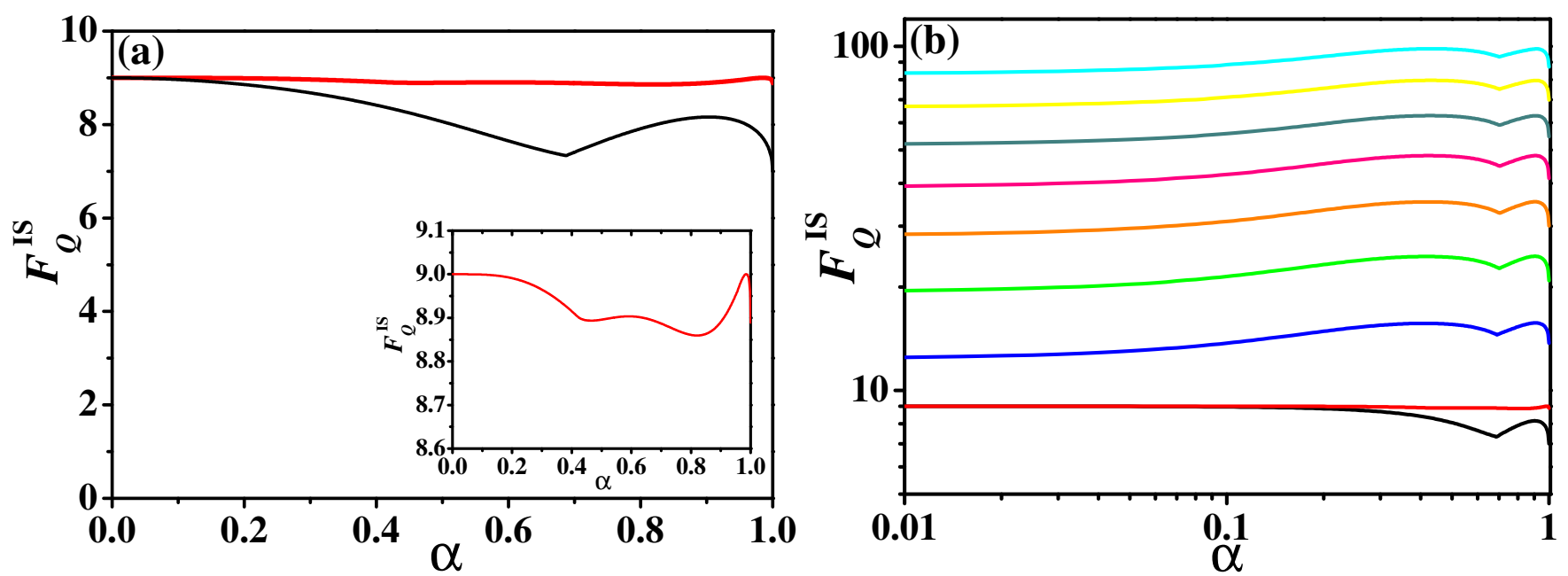

Fig. 1: (color online) The QFI of 3-qubit superposition state with respect to ratio $\alpha$. a) Comparison of QFI under local operation ( $\varepsilon=0$, black line) and nonlocal operation $(\varepsilon=1$, red line), the inset shows the detailed changes. b) The trend of QFI with respect to the increasing strength $\varepsilon$. From the bottom to top, the colorful lines denote the $\varepsilon$ from 0 to 8 , respectively.

are many a permutation needed to be calculated in a large-scale quantum state. However, due to symmetry of the superposition state Eq. (2), all the terms required for QFI are same under the exchange of qubits. As a result we just need to calculate any one of these permutations and then assemble them for final QFI. After calculations we find the explicit form of the first-order moment of interactive qubits are different when the number of qubits is $N<6$. Next we take 3-qubit W and GHZ as a paradigm to elaborate it.

At the beginning we present the QFI of $N$-qubit superposition state in Ising model,

$$
\begin{aligned}
\frac{F_{Q}^{\mathrm{IS}}}{N} & =1-N\left\langle\sigma_{n}^{(i)}\right\rangle^{2}+(N-1)\left\langle\sigma_{n}^{(i)} \sigma_{n}^{(j)}\right\rangle \\
& +\varepsilon\left(2\left\langle\sigma_{n}^{(i)}\right\rangle-N\left\langle\sigma_{n}^{(i)} \sigma_{n}^{(j)}\right\rangle\left\langle\sigma_{n}^{(i)}\right\rangle+(N-2)\left\langle\sigma_{n}^{(i)} \sigma_{n}^{(j)} \sigma_{n}^{(k)}\right\rangle\right) \\
& +\frac{\varepsilon^{2}}{4}\left(1+2\left\langle\sigma_{n}^{(i)} \sigma_{n}^{(j)}\right\rangle+(N-3)\left\langle\sigma_{n}^{(i)} \sigma_{n}^{(j)} \sigma_{n}^{(k)} \sigma_{n}^{(l)}\right\rangle-N\left\langle\sigma_{n}^{(i)} \sigma_{n}^{(j)}\right\rangle^{2}\right) .
\end{aligned}
$$

Given the 3-qubit $\mathrm{W}$ and GHZ superposition state,

$$
\left|\psi_{3}\right\rangle=\frac{\alpha}{\sqrt{3}}(|001\rangle+|010\rangle+|010\rangle)+\frac{\beta}{\sqrt{2}}(|000\rangle+|111\rangle)
$$

the averaged terms are calculated as

$$
\begin{aligned}
\left\langle\sigma_{n}^{(i)}\right\rangle & =\frac{2 \alpha \beta}{\sqrt{6}} a-\frac{\alpha^{2}}{3} c \\
\left\langle\sigma_{n}^{(i)} \sigma_{n}^{(j)}\right\rangle & =\left(\frac{2 \alpha \beta}{\sqrt{6}}+\frac{2 \alpha^{2}}{3}\right) a^{2}-\frac{4 \alpha \beta}{\sqrt{6}} a c+\left(-\frac{2 \alpha \beta}{\sqrt{6}}+\frac{2 \alpha^{2}}{3}\right) b^{2}+\left(\beta^{2}-\frac{\alpha^{2}}{3}\right) c^{2} \\
\left\langle\sigma_{n}^{(i)} \sigma_{n}^{(j)} \sigma_{n}^{(k)}\right\rangle & =a^{3} \beta^{2}+c^{3} \alpha^{2}+3 a^{2} c\left(\frac{2 \alpha \beta}{\sqrt{6}}-\frac{2 \alpha^{2}}{3}\right)-3 a b^{2} \beta^{2}+3 a c^{2} \frac{2 \alpha \beta}{\sqrt{6}}+3 b^{2} c\left(-\frac{2 \alpha \beta}{\sqrt{6}}-\frac{2 \alpha^{2}}{3}\right) .
\end{aligned}
$$

Replacing above equations into Eq. (9), the QFI of 3-qubit superposition state is obtained. Similarly, we have access to the QFI of 4,5-qubit superposition state. 


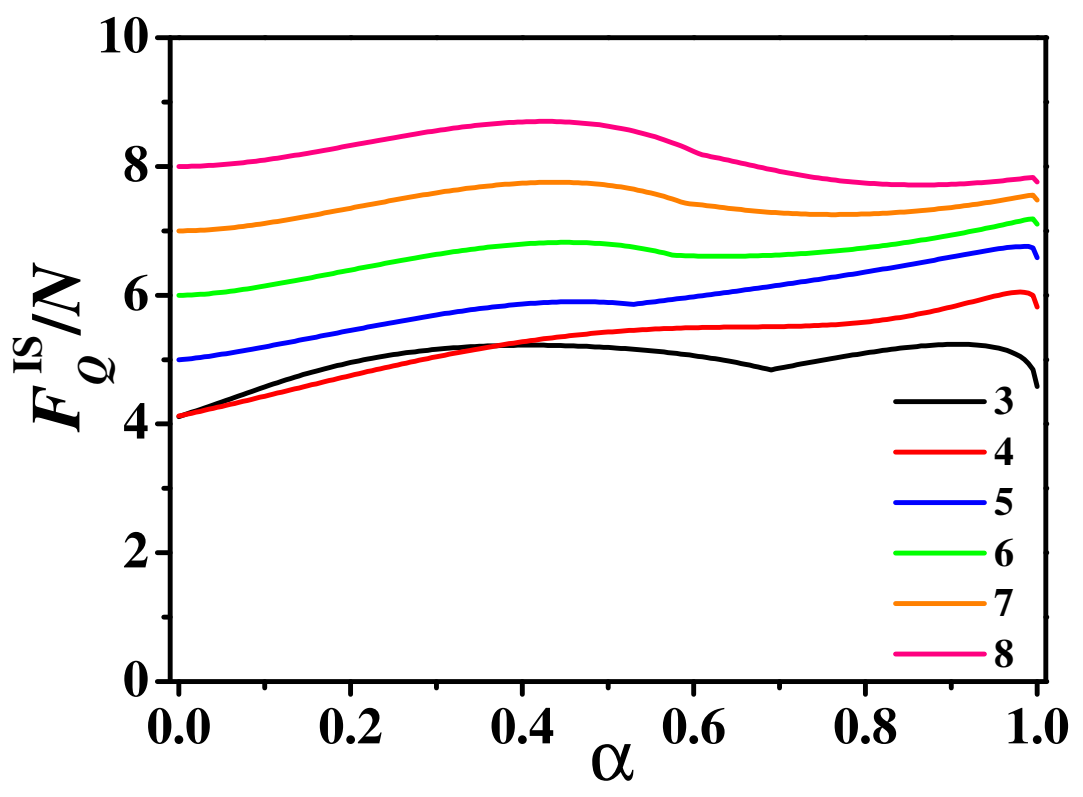

Fig. 2: (color online) The QFI of $N=3, \ldots, 8$-qubit superposition state with respect to $\alpha$ in Ising model, and the interaction strength $\varepsilon=2$. With the increase of number of qubits, the optimal $\alpha$ for maximal QFI is changed form close to 1 to the middle region.

As the number of qubits increases larger than 5 , a general formula is found to represent the QFI of superposition state $\left|\psi_{N}\right\rangle$. The averaged terms are calculated as

$$
\begin{aligned}
\left\langle\sigma_{n}^{(i)}\right\rangle & =\frac{2-N}{N} c \alpha^{2}+\sqrt{\frac{2}{N}} a \alpha \beta \\
\left\langle\sigma_{n}^{(i)} \sigma_{n}^{(j)}\right\rangle & =\frac{2 \alpha^{2}}{N}\left(a^{2}+b^{2}\right)-2 \sqrt{\frac{2}{N}} a c \alpha \beta+c^{2}\left(\frac{N-4}{N} \alpha^{2}+\beta^{2}\right) \\
\left\langle\sigma_{n}^{(i)} \sigma_{n}^{(j)} \sigma_{n}^{(k)}\right\rangle & =-\frac{6 c \alpha^{2}}{N}\left(a^{2}+b^{2}\right)+\frac{6-N}{N} c^{3} \alpha^{2}+3 \sqrt{\frac{2}{N}} a c^{2} \alpha \beta \\
\left\langle\sigma_{n}^{(i)} \sigma_{n}^{(j)} \sigma_{n}^{(k)} \sigma_{n}^{(l)}\right\rangle & =\frac{12 c^{2} \alpha^{2}}{N}\left(a^{2}+b^{2}\right)-4 \sqrt{\frac{2}{N}} a c^{3} \alpha \beta+\frac{c^{4}}{N}\left((N-8) \alpha^{2}+N \beta^{2}\right),
\end{aligned}
$$

and the QFI of $N$-qubit superposition state is achieved by substituting above equations into Eq. (9). Due to the lengthy of the formula, it is not shown here.

In Fig. 1, we numerically show the QFI of 3-qubit superposition state $\left|\psi_{3}\right\rangle$ with respect to $\alpha$. It is interesting that the QFI is almost unchanged as $\varepsilon=1$ in Fig. 1a, which implies that the superposition state can be used to achieve the conventional Heisenberg limit in all $\alpha$ region. This maybe helpful to the experimental research. Meanwhile, we find the QFI under local operation $(\varepsilon=0)$ is in line with the result shown in Ref. [36], where the RMQFI is plotted with respect to $\alpha$. In Fig. 1b, with the increase of $\varepsilon$, the trends of QFI are similar and there always exists a turning point for QFI, where the $\alpha$ is around 0.7 .

For the case of $N$-qubit superposition state, we present the QFI with respect to $\alpha$ in Fig. 2, in which the interaction strength is same and $\varepsilon=2$. It is easy to found that with the increasing qubits involved, the ratio $\alpha$ for the maximal QFI is changed from left side (close to 1 ) to the middle region. Meanwhile, the position of $\alpha$ for the turning point appeared in the 3-qubit superposition state is changed, as it is shown by the blue line and other colorful lines. Additionally, there also exists a sudden change of QFI for all $N$-qubit superposition state and the $\alpha$ is close to 1 .

\subsection{QFI of $N$-qubit superposition state in LMG model}

In this section, we consider the fully-connected nonlocal operation, Lipkin-Meshkov-Glick model, originated from the nuclear physics and used to describe many-body problem approximation [41]. Likewise, $\mathbf{m} \cdot \mathbf{n}=1$ and $V_{i j}=\varepsilon$, the QFI of $N$-qubit 


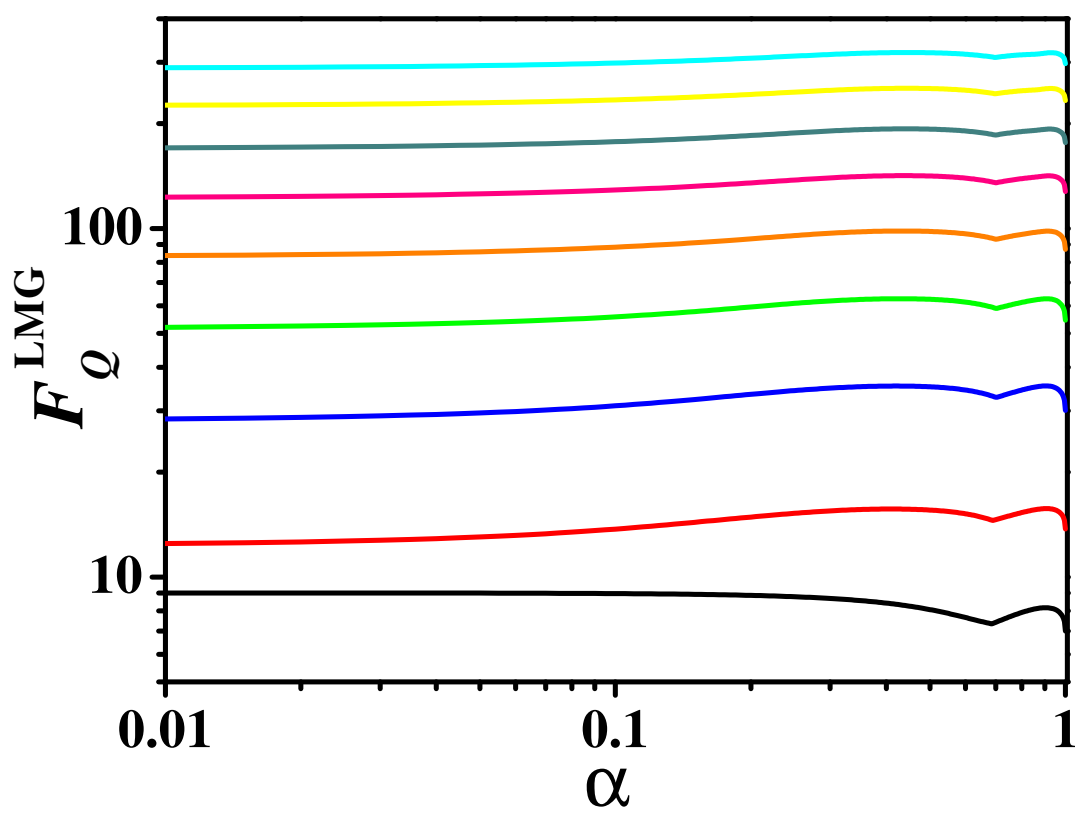

Fig. 3: (color online) The QFI of 3-qubit superposition state with respect to $\alpha$ in LMG model, where the colorful lines respectively represent the interaction strength $\varepsilon=0, \ldots, 8$ (from bottom to top).

Table 1: Turning point (TP) for QFI of 3-qubit superposition state with respect to $\varepsilon$ in Ising model and LMG model.

\begin{tabular}{llllllllll}
\hline \hline$\varepsilon$ & 0 & 1 & 2 & 3 & 4 & 5 & 6 & 7 & 8 \\
\hline TP (Ising) & 0.687 & null & 0.690 & 0.700 & 0.703 & 0.703 & 0.703 & 0.702 & 0.701 \\
\hline TP (LMG) & 0.687 & 0.690 & 0.703 & 0.703 & 0.701 & 0.700 & 0.699 & 0.698 & 0.697 \\
\hline \hline
\end{tabular}

superposition state is expressed as

$$
\begin{aligned}
\frac{F_{Q}^{\mathrm{LMG}}}{N} & =1-N\left\langle\sigma_{n}^{(i)}\right\rangle^{2}+(N-1)\left\langle\sigma_{n}^{(i)} \sigma_{n}^{(j)}\right\rangle \\
& +(N-1) \varepsilon\left(2\left\langle\sigma_{n}^{(i)}\right\rangle-N\left\langle\sigma_{n}^{(i)} \sigma_{n}^{(j)}\right\rangle\left\langle\sigma_{n}^{(i)}\right\rangle+(N-2)\left\langle\sigma_{n}^{(i)} \sigma_{n}^{(j)} \sigma_{n}^{(k)}\right\rangle\right) \\
& +\frac{(N-1) \varepsilon^{2}}{4}\left(2+(4 N-8)\left\langle\sigma_{n}^{(i)} \sigma_{n}^{(j)}\right\rangle-\left(N^{2}-N\right)\left\langle\sigma_{n}^{(i)} \sigma_{n}^{(j)}\right\rangle^{2}+(N-2)(N-3)\left\langle\sigma_{n}^{(i)} \sigma_{n}^{(j)} \sigma_{n}^{(k)} \sigma_{n}^{(l)}\right\rangle\right),
\end{aligned}
$$

where all the interactive terms required are same under the exchange of qubits.

Replacing previous Eqs. (11,12,13) into Eq. (14), we have access to the QFI of 3-qubit superposition state in LMG model. In Fig. 3, we numerically plot the QFI with respect to ratio $\alpha$. Obviously it looks like an enlarged version of the Fig. 1b, except for the case $\varepsilon=1$. Because all the interactive terms are contributed to final QFI, it gives a larger QFI in LMG model than Ising model. Similarly, there still exists a turning point for all $N$-qubit superposition state.

To describe it clearly, we in detail record the value of turning points (tps) from both models in Table. 1, where the results are numerically obtained under the different $\varepsilon$. It shows that the tps for 3-qubit superposition state is relatively stable and the ratio $\alpha$ is around 0.7 , except for the case of $\varepsilon=1$ in Ising model.

We then investigate the QFI of $N$-qubit superposition state in LMG model. Under the same interaction strength $\varepsilon=2$, the QFI of $N=3, \ldots, 8$ qubits superposition state with respect to $\alpha$ are shown in Fig. 4. Apparently, with the increase of the number of qubits, the QFI is largely enhanced and the turn points are almost disappeared. However, the sudden change of QFI is still displayed when the ratio $\alpha$ approaches 1 , and this indicates that if one chooses the large-scale superposition state for quantum metrology, the optimal $\alpha$ for the maximal QFI is close to 1 but not 1 . 


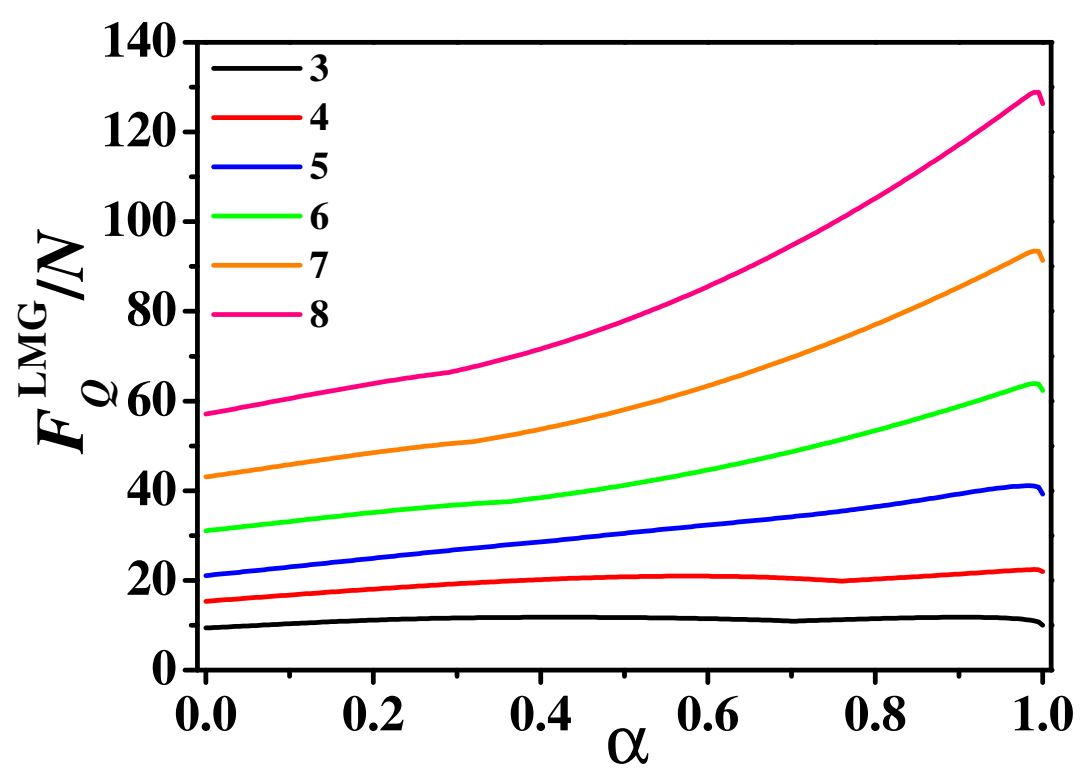

Fig. 4: (color online) The QFI of $N=3, \ldots, 8$-qubit superposition state with respect to $\alpha$ in LMG model, where the interaction strength $\varepsilon=2$. With the increasing of number of qubits, the optimal $\alpha$ for maximal QFI is close to 1 but not 1 .

\section{Conclusions}

In summary, we have studied the QFI of $N$-qubit W and GHZ superposition state under nonlocal operation. By utilizing the formula to calculate QFI under two-body nonlocal operation, we explicitly present the QFI of superposition state in Ising model and LMG model. The results show that the conventional fixed QFI is evidently changed, and becomes a parameter-controlled QFI. This greatly facilitates the quantum precision measurement and other quantum information processing. To learn more about the QFI in both models, we in detail investigate the QFI of 3-qubit superposition state with respect to ratio $\alpha$ and interaction strength $\varepsilon$. There always exists a turning point for QFI in both models and it is around $\alpha=0.7$. Meanwhile, for $\varepsilon=1$ in Ising model, the well-known Heisenberg limit is almost attained for all $\alpha$. Then we compare the QFI of $N$-qubit superposition state under the same interaction strength, and find the position of $\alpha$ for the maximal QFI is different in both models. With the increase of the number of qubits, the optimal $\alpha$ in Ising model is changed from close to 1 to the middle region, whereas it is unchanged in LMG model. We hope that our work promotes the development of nonlinear quantum metrology, and further provides help for quantum information processing.

\section{Acknowledgments}

This work was supported by the Scientific and Technological Innovation Programs of Higher Education Institutions in Shanxi (Grant No. 2020L0512), the 1331 Program of Taiyuan Normal University (Grant No. I0190364) and the Doctoral Scientific Research Foundation of Taiyuan Normal University.

\section{Declaration of interests}

The authors declare no competing interests.

\section{References}

1. C.W. Helstrom, Quantum Detection and Estimation Theory. Academic Press, New York (1976).

2. A.S. Holevo, Probabilistic and Statistical Aspects of Quantum Theory. North Holland, Amsterdam (1982).

3. W.K. Wootters, Statistical distance and Hilbert space. Phys. Rev. D. 23, 357 (1981).

4. S.L. Braunstein and C.M. Caves, Statistical Distance and the Geometry of Quantum States. Phys. Rev. Lett. 72, 3439 (1994).

5. R.A. Fisher, Theory of statistical estimation. Math. Proc. Camb. Philos. Soc. 22,700-725 (1925).

6. B.R. Frieden, Science from Fisher Information: A Unification. Cambridge University Press, New York (2004).

7. V. Giovannetti, S. Lloyd and L. Maccone, Quantum Metrology. Phys. Rev. Lett. 96, 010401 (2006). 
8. Giovannetti, V., Lloyd, S., and Maccone, L.: Advances in quantum metrology. Nat. Photon. 5, 222-229 (2011)

9. L. Pezzé, A. Smerzi, M.K. Oberthaler, R. Schmied, P. Treutlein, Non-classical states of atomic ensembles: fundamentals and applications in quantum metrology. Rev. Mod. Phys. 90, 035005 (2018).

10. R. Horodecki, P. Horodecki, M. Horodecki, K. Horodecki, Quantum entanglement. Rev. Mod. Phys. 81, 865 (2009).

11. O. Gühne and G. Tòth, Entanglement detection. Phys. Rep. 474, 1 (2009).

12. P. Zanardi, M.G.A. Paris, L.C. Venuti, Quantum criticality as a resource for quantum estimation. Phys. Rev. A 78, 042105 (2008).

13. J. Ma and X.G. Wang, Fisher information and spin squeezing in the Lipkin-Meshkov-Glick model. Phys. Rev. A 80, 012318 (2009).

14. T.L. Wang, L.N. Wu, W. Yang, G.R. Jin, N. Lambert and F. Nori, Quantum Fisher information as a signature of the superradiant quantum phase transition. New J. Phys. 16, 063039 (2014).

15. P. Hauke, M. Heyl, L. Tagliacozzo, P. Zoller, Measuring multipartite entanglement through dynamic susceptibilities. Nat. Phys. 12, 778782 (2016).

16. M. Gabbrielli, A. Smerzi, and L. Pezzé, Multipartite Entanglement at Finite Temperature. Sci. Rep. 8, 15663 (2018).

17. Cramér, H.: Mathematical methods of statistics. Princeton University, Princeton (1946)

18. C. Helstrom, Minimum mean-squared error of estimates in quantum statistics, Phys. Lett. A 25, 101 (1967).

19. X.M. Lu, S. Yu, C.H. Oh, Robust quantum metrological schemes based on protection of quantum Fisher information, Nat. Commun. 6 , $7282(2015)$.

20. Z.M. Huang, Protecting quantum Fisher information in curved space-time, Eur. Phys. J. Plus 131, 102 (2018).

21. L. Pezzè and A. Smerzi, Entanglement, Nonlinear Dynamics, and the Heisenberg Limit. Phys. Rev. Lett. 102, 100401 (2009).

22. P. Hyllus, W. Laskowski, R. Krischek, C. Schwemmer, W. Wieczorek, H. Weinfurter, L. Pezzè and A. Smerzi, Fisher information and multiparticle entanglement. Phys. Rev. A 85, 022321 (2012).

23. G. Tòth, Multipartite entanglement and high-precision metrology. Phys. Rev. A 85, 022322 (2012).

24. Y. Li and P.F. Li, Detection of genuine N-Qubit W state, GHZ state and Twin-Fock state via Quantum Fisher information. Phys. Lett. A 384, 126413 (2020).

25. R.C. Almeida and P. Hauke, From entanglement certification with quench dynamics to multipartite entanglement of interacting fermions. Phys. Rev. Res. 2, L032051 (2021).

26. Z.Z. Qin, M. Gessner, Z.H. Ren, X.W. Deng, D.M. Han, W.D. Li, X.L. Su, A. Smerzi and K.C. Peng, Characterizing the multipartite continuous-variable entanglement structure from squeezing coefficients and the Fisher information. npj Quantum Inf. 5, 3 (2019).

27. Z.H. Ren, W.D. Li, A. Smerzi, M. Gessner, Metrological Detection of Multipartite Entanglement from Young Diagrams. Phys. Rev. Lett. 126, 080502 (2021).

28. B. Lücke, M. Scherer, J. Kruse, L. Pezzè, F. Deuretzbacher, P. Hyllus, O. Topic, J. Peise, W. Ertmer, J. Arlt, L. Santos, A. Smerzi, C. Klempt, Twin Matter Waves for Interferometry Beyond the Classical Limit. Science 334, 773 (2011).

29. H. Strobel, W. Muessel, D. Linnemann, T. Zibold, D.B. Hume, L. Pezzè, A. Smerzi and M.K. Oberthaler, Fisher information and entanglement of non-Gaussian spin states. Science 345, 424 (2014).

30. Czekaj, Ł., Przysiężna, A., Horodecki, M. and Horodecki, P.: Quantum metrology: Heisenberg limit with bound entanglement, Phys. Rev. A 92, 062303 (2015).

31. J. Smith, A. Lee, P. Richerme, B. Neyenhuis, P.W. Hess, P. Hauke, M. Heyl, D.A. Huse and C. Monroe, Many-body localization in a quantum simulator with programmable random disorder. Nat. Phys. 12, 907-911 (2016).

32. J.G. Bohnet, B.C. Sawyer, J.W. Britton, M.L. Wall, A.M. Rey, M. Foss-Feig, J.J. Bollinger, Quantum spin dynamics and entanglement generation with hundreds of trapped ions. Science 352, 1297-1301 (2016).

33. G. Mathew, et al., Experimental realization of multipartite entanglement via quantum Fisher information in a uniform antiferromagnetic quantum spin chain. Phys. Rev. Res. 2, 043329 (2015).

34. L. Pezzè, Y. Li, W.D. Li and A. Smerzi, Witnessing entanglement without entanglement witness operators. Proc. Natl. Acad. Sci. 113, 11459-11464 (2016).

35. P. Walther, K.J. Resch, and A. Zeilinger, Local Conversion of Greenberger-Horne-Zeilinger States to Approximate W States. Phys. Rev. Lett. 94, 240501 (2005).

36. O. Fatih, A. Azmi Ali, B. Sinan, Y. Can, Quantum Fisher Information of N Particles in the Superposition of W and GHZ States. Int. J. Theor. Phys. 52, 2977 (2013).

37. G.M. Yuan, W. Song, M. Yang, D.C. Li, J.L. Zhao, and Z.L. Cao, Monogamy relation of multi-qubit systems for squared Tsallis-q entanglement. Sci. Rep. 6, 28719 (2016).

38. X.F. Jin, L.Z. Jiang, and X.Y. Chen, Entanglement properties of GHZ and W superposition state and its decayed states. Chin. Phys. B 30, 060301 (2021).

39. U. Khalid, J. Rehman and H. Shin, Metrologically resourceful multipartite entanglement under quantum many-body effects. Quantum Sci. Technol. 6, 025007 (2021).

40. E. Ising, Contribution to the Theory of Ferromagnetism. Z. Phys. 31, 253-258 (1925).

41. H.J. Lipkin, N. Meshkov and A. Glick, Validity of many-body approximation methods for a solvable model: (I). Exact solutions and perturbation theory. Nucl. Phys. 62, 188 (1965).

42. Y. Li, L. Pezzè, W. Li, A. Smerzi, "Sensitivity bounds for interferometry with Ising Hamiltonians”, Phys. Rev. A 99, 022324 (2019). 